\title{
NUMERICAL STUDY AND EXPERIMENTAL INVESTIGATION OF AN ELECTROHYDRODYNAMIC DEVICE FOR INERTIAL SENSING
}

\author{
Thu-Hang Nguyen ${ }^{1}$, Ngoc Van Tran ${ }^{2}$, Thien Xuan Dinh ${ }^{3}$, Canh-Dung Tran ${ }^{4}$, Van Thanh Dau ${ }^{5 *}$ \\ Trinh Duc Chu ${ }^{1}$, Hai Nguyen Hoang ${ }^{l}$, and Tung Thanh Bui ${ }^{1 * *}$ \\ ${ }^{1}$ University of Engineering and Technology, Vietnam National University, Hanoi, VIETNAM \\ ${ }^{2}$ Institute of Missile - Academy of Military Science and Technology, VIET NAM \\ ${ }^{3}$ Explosion Research Institute Inc., R\&D Division, Tokyo, JAPAN \\ ${ }^{4}$ School of Mechanical and Electrical Engineering, University of Southern Queensland, Queensland \\ AUSTRALIA

\section{${ }^{5}$ School of Engineering and Built Environment, Griffith University, Queensland, AUSTRALIA}

\begin{abstract}
We present a multi-physics simulation associated with experimental investigation for an electrohydrodynamic gyroscope based on ion wind corona discharge. The present device consisting of multiple point-ring electrodes generates a synthetic jet flow of ions for inertial sensing applications. Meanwhile the residual charge of jet is neutralized by an external ring electrode to guarantee the ion wind stable while circulating inside the device's channels. The working principle including the generation and then circulation of jet flow within the present device is firstly demonstrated by a numerical simulation and the feasibility and stability of the device are then successfully investigated by experimental work. Results show owing to the ion wind corona discharge based approach associated with new configuration, the present device is robust and consumes low energy.
\end{abstract}

\section{KEYWORDS}

Multiphysics simulation, ionic wind corona discharge, inertial sensing, gyroscope, electrohydrodynamic

\section{INTRODUCTION}

Jet flow gyroscope is an inertial sensing device utilizing laminar flow to detect angular rate by the induced Coriolis force. In these sensors, generating a stable jet flow is one of the most challenging technical barriers but interesting, yielding several achieved approaches [1]-[7] . One of the latest technique is based on the corona-discharge principle where a jet flow is created by applying a high-voltage between a discharge electrode and a reference electrode [8]. This approach does not require any vibrating component, and possesses several advantages including lower cost and energy consumption; tidy and light; and simple operation, compared with those by other methods using air pump [9] or oscillating piston [10].

For closed corona discharge based systems, for example, systems using the configuration of pin-to-ring electrodes, pin plays the role of the corona electrode and ring as the collector. For such configuration, ion wind is partially neutralized when reaching to the ring surface, and the circulatory ion wind inevitably result in residual electric charge. This yields a reversed electrical field which damages the corona discharge process. Although this issue can be fixed using embedded neutralizing components or grounding the system, such configurations are fairy complicated in designing and manufacturing, and there were very limited literature and report so far.

The system presented in this paper is based on corona discharge ion wind but using multiple pairs of point-ring electrodes and is developed for inertial sensing applications [11]-[13]. The key of this enhancement is how to arrange ring electrodes in the new configuration to neutralize the residual charge, thus capable of circulating a stable ion flow inside the system. In this work, we represent the multiphysics simulation and in-depth analysis of jet flow in 3D space using OpenFOAM, together with experimental investigation to validate the feasibility and reliability of the present approach.

\section{MECHANISM AND MODELLING OF DEVICE}

Figure 1 presents a one-third of an axial symmetric fluidic network, consisting of three cylindrical ion wind chambers which connect together before being linked with a working/sensing chamber via a nozzle at the device center where hotwires are installed. The dimensions (diameter $\times$ length) of the ion wind and working cylindrical chambers are $5 \mathrm{~mm} \times 10 \mathrm{~mm}$ and $12 \mathrm{~mm} \times$ $15 \mathrm{~mm}$, respectively. For each ion wind chamber, a pair of pin-ring electrodes is installed and plays the role of ion wind actuator. Pin, a cylindrical electrode of $0.4 \mathrm{~mm}$ diameter with a spherical tip of $80 \mu \mathrm{m}$ radius, is placed at $5 \mathrm{~mm}$ from the corresponding ring electrode. At the downstream of working chamber, one bigger ring electrode is installed to neutralize the residual charge caused by ion winds.

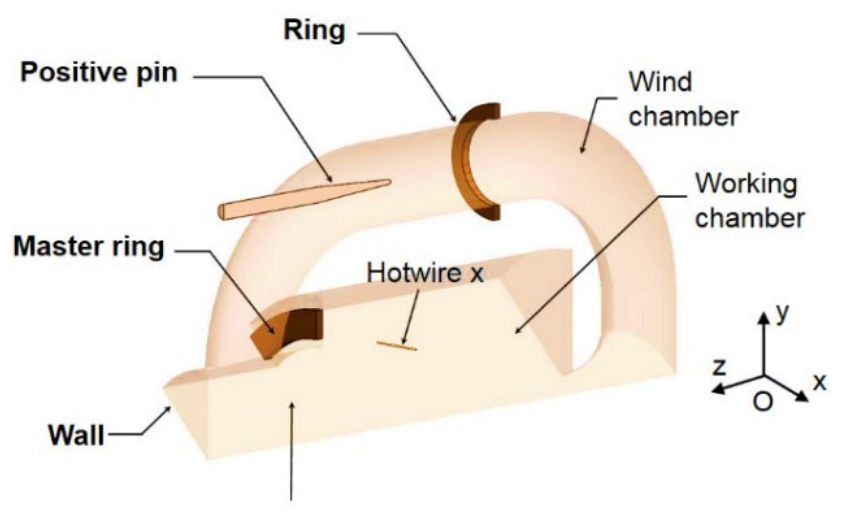

Symmetry plane

Figure 1. A schema of the device: conceptual model and mechanism. 
Model shown in Fig. 2 is used to analyze the flow characteristics within the system. A multiphysics simulation of the model relating to (i) an electrical field which induces the migration of ion wind through the inter-electrode region and its interaction with air flow inside chambers; and (ii) the dynamic behavior of air flow, is carried out. Charged ion winds generated by pin electrodes moving under the effect of electric field drift air flow which, in turn, redistribute themselves across the system domain.

Neglecting the conduction and diffusion of ions going through air and considering the steady state, the charge density $q$ of ion wind caused by the ion drift $(\mu \vec{E} q)$ and ion convection $(\vec{U} q)$ are governed by the equation of charge conservation and Gauss' law, respectively as follows

$$
\begin{gathered}
\nabla \cdot(\mu \vec{E} q+\vec{U} q)=0, \\
\nabla \cdot(\nabla \emptyset)=-q / \varepsilon_{0},
\end{gathered}
$$

where $\mu=1.6 \times 10^{-4} \mathrm{~m}^{2} \mathrm{~V}^{-1} \mathrm{~s}^{-1}$ is the ion mobility; $\vec{U}$ the velocity of air drifted by the charged ion motion and $\epsilon_{0}=8.854 \times 10^{-12} \mathrm{CV}^{-1} \mathrm{~m}^{-1}$ the permittivity of air. Electric field $\vec{E}$ is a function of the electric potential and defined as $\vec{E}=-\nabla V$.

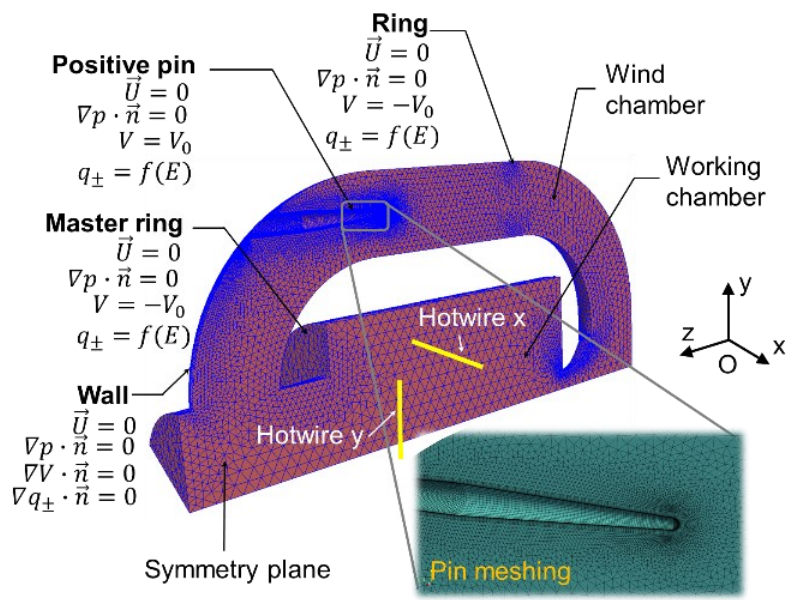

Figure 2. Numerical simulation: Geometrical model, meshing and boundary conditions.

Boundary condition on electrodes' surface is set up by the charge density $q_{s}$ by the corona discharge. Given as a function of the discharge current I, $q_{s}$ is determined from the I-V experimental curve as follows

$$
q_{s}=I /\left(\mu E_{\text {on }} A\right)
$$

where $\mathrm{A}$ is the total area of electrodes generating the electric field whose magnitude is greater than an onset of Eon $=3.23 \times 106 \mathrm{~V} / \mathrm{m}$ [14]. The potential applied on the pin and ring electrodes are $\mathrm{V}$ and 0 , respectively. The Townsend I-V relationship which is efficient for point-toring configuration as demonstrated in [15], [16]

Inside channels, air flow, a self-generating flow system of incompressible and steady state, is governed by the Navier-Stokes and the continuity equations, respectively as:

$$
\begin{aligned}
\nabla \cdot(\vec{U} \vec{U})-\nabla \cdot(v \nabla \vec{U}) & =-\nabla p+\frac{f_{e}}{\rho}-2 \rho \vec{\omega} \times \vec{U}, \\
\nabla \cdot \vec{U} & =0,
\end{aligned}
$$

where term $2 \rho \vec{\omega} \times \vec{U}$ is the Coriolis apparent acceleration by the rotating frame with the angular velocity $\vec{\omega} ; p$ pressure; $v=15.7 \times 10^{-3} \mathrm{~m}^{2} \cdot \mathrm{s}^{-1}$ the air viscosity; $\rho=$ $1.2041 \mathrm{~kg} \cdot \mathrm{m}^{-3}$ the air density; $f_{e}$ the electromagnetic force acting on the corona and consisting of the Coulomb, permittivity gradient, dielectrophoretic and electrocostriction forces. In this work, since air flow with a given temperature gradient is considered, the last three forces are neglected and thus, the electromagnetic force includes only the Coulomb one and given by

$$
f_{e}=q \vec{E} .
$$

Since air flow is a self-generating system, no particular flow condition was applied except the nonslip condition on the electrodes and the channels' wall.

Boundary conditions and domain meshing are detailed in Fig. 2. The solution of Eqs. (1)-(6) together with boundary conditions is obtained using our solver which was developed using OpenFOAM based finite volume method [17].

\section{RESULTS AND DISCUSSION Simulation results}

Ion wind generated by the corona actuator between pin-ring electrodes and migrating through channels is numerically illustrated in Fig. 3a\&b for two cases where the device is at rest and rotates about $x$ axis with a speed of $100 \mathrm{rpm}$. The simulation results demonstrated that a jet flow of ions generated by the corona from pin tips recirculates to pins through three subsidiary channels to define an air flow network inside the device.

(a)
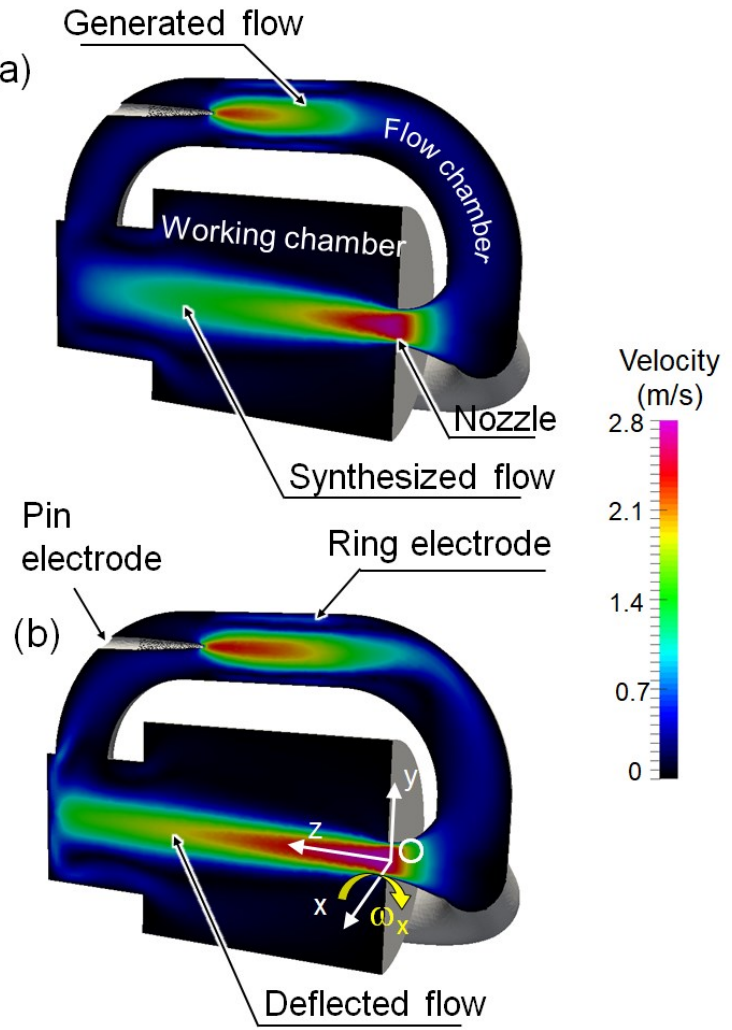

Figure 3. Simulating results: Cut view of velocity contour demonstrates the generation of jet flow and its circulation inside the device in two cases: (a) device at rest and (b) device rotates with a given speed. 
In both cases, the simulating observation by Fig. 4 found that the velocity profile reduces at farther distance (L) counted from the nozzle but the jet form of the profile is maintained. Figure 4 illustrates the velocity profiles $U_{z}$ plotted with respect to the $x$ direction (denoted by $U_{z / x}$ ) and respect to the $y$ direction (denoted by $U_{z / y}$ ) at several different positions $L=1,3,5,7,9,11 \mathrm{~mm}$ from the nozzle while the device rotates with an angular velocity of $100 \mathrm{rpm}$ about $X$ axis. It can be seen that ion wind flow deflects around the $y$ direction; leading to the asymmetric velocity profile $U_{z / y}$. This asymmetry acts as the input for the hotwire placed inside the working chamber.
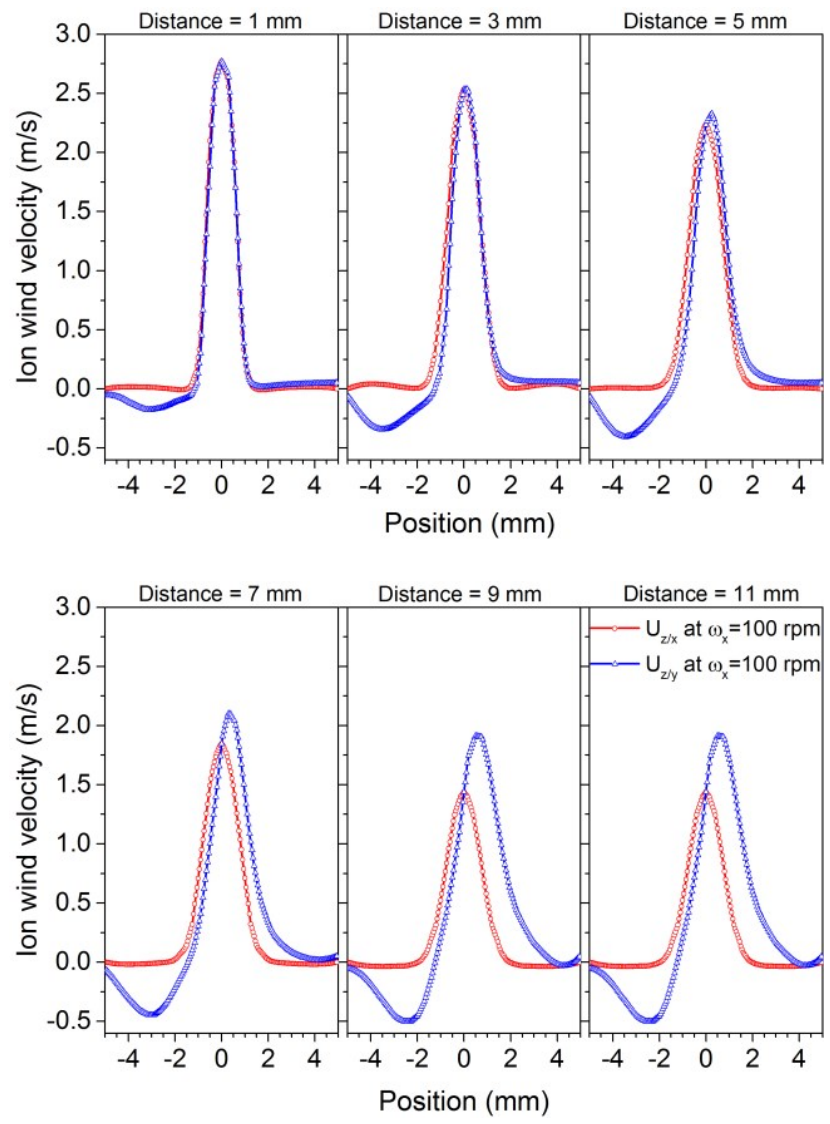

Figure 4: Simulation results: Velocity profiles $U_{z / x}$ (red line) and $U_{z / y}$ (blue line) at five positions $(L=1,3,5,7,9$ and $11 \mathrm{~mm}$ counted from nozzle to the left side) in working chamber when the device rotates with angular rates $\omega_{x}=100 \mathrm{rpm}$

The velocity $U$ of ion wind is estimated from the discharge current $I$ as:

$$
U=k \sqrt{I / \rho \mu}
$$

Let $\beta$ be the gradient of the velocity of air flow going through the plane of sensing element, the deflection of air flow $\left(\Delta U_{\omega}\right)$ can be converted into a variation of air flow velocity as follows:

$$
\Delta U_{\omega}=\left(\beta \omega L^{2} / \mathrm{k}\right) \sqrt{\rho \mu / I}
$$

By a constant electric current, heat is transferred through hot wires by conduction, convection and radiation regimes but not radiation because the working temperature of sensing elements is not too high in this work. In continuous medium, heat conduction is determined by Fourier's law:

$$
\mathrm{q}=-\mathrm{k} \nabla \mathrm{T} \text {, }
$$

where $\mathrm{q}$ is the convective heat flux, $\mathrm{k}$ thermal conductivity, $\mathrm{T}$ the temperature.

The deflection of ion wind due to Coriolis force leads to the asymmetric temperature characteristic of two hot wires. Temperature of the hot wires on the side where ion wind flows through is lower than one of hot wires located on another side.

$$
\delta \mathrm{T}=\frac{1}{\mathrm{~V}_{\mathrm{hw} 2}} \int_{\mathrm{V}_{\mathrm{hw} 2}} \mathrm{TdV}-\frac{1}{\mathrm{~V}_{\mathrm{hw} 1}} \int_{\mathrm{V}_{\mathrm{hw} 1}} \mathrm{TdV}
$$

where $\delta \mathrm{T}$ is the temperature difference between two hot wires, $\mathrm{V}_{\mathrm{hw} 1}, \mathrm{~V}_{\mathrm{hw} 2}$ are volumes of hot wires 1 and 2 , respectively and $\mathrm{T}$ the temperature of each volume unit.

\section{Experimental investigation}

Experimental investigation is carried out using a prototype which was fabricated by $3 \mathrm{D}$ printing Objet500 Connex3 (Stratasys Ltd.). Three pairs of SUS pin - ring electrodes whose dimensions fit the simulation parameters are connected to a high voltage source (Glassman EH10R10). Four tungsten hotwires are installed in the main chamber. The device together with air at room condition is packed and sealed.

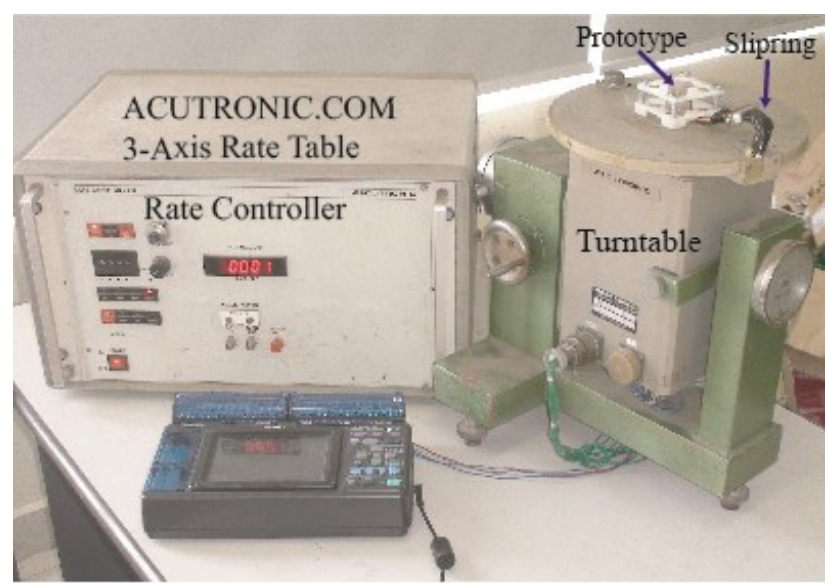

Figure 5: The fabricated prototype is mounted on a standard Acutronic turntable.

The performance of the fabricated sensor prototype is evaluated using a standard Acutronic turntable. The experiment with equipment and measuring devices is shown in Figure 5. The prototype mounted on a turntable which can be set at different angular speeds by the rate controller of Acutronic system. The speed of the turntable is used as the reference output for the experiment.

As illustrated in Figs $6 \& 7$, the measured results of the angular velocity sensor based on corona discharge and the indication of the standard Acutronic turntable are completely equivalent. The difference of results measured by theofwo devices is no more than $1 \%$., demonstrating that the presented sensor has a good repeatability, the sensor speed is almost no different in both the acceleration direction (on the left side of graph) and the deceleration direction (on the right side of the graph). 


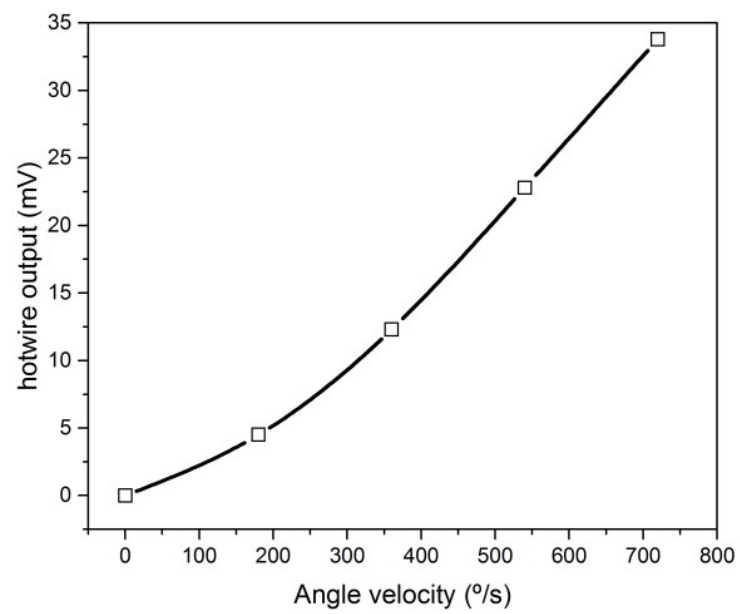

Figure 6: Output voltage measured on hotwire plotted versus the angular velocity of turntable.

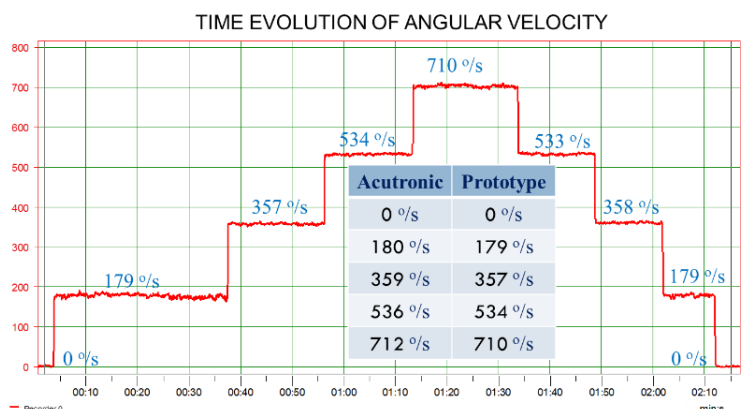

Figure 7: Graph of sensor's angular rate with standard Acutronic turntable. Table (inset) shows the Acutronic's input as the reference angular rate versus our sensor's output during the test. Our device showed good performance and high stability. The output (in $\%$ is converted from the measured sensivitivy in Fig. 6)

\section{CONCLUSIONS}

We have presented the multiphysics simulation that serves as the design tool for an electrohydrodynamic gyroscope and experimental validation of the fabricated prototype. Firstly, jet flow of ions generated by a configuration of pin-ring electrodes was demonstrated by the numerical simulation. Results by the numerical simulation are useful in parameter optimization of the device. Secondly, the feasibility and stability of the present approach based device are successfully investigated by experimental works. For inertial applications, the present electrohydrodynamic device features (i) the robustness owing not to require any vibrating component, and (ii) low energy consumption inherited from the corona ion wind approach.

\section{ACKNOWLEDGEMENT}

This work has been supported/partly supported by VNU University of Engineering and Technology under project number CN20.31

\section{REFERENCE}

[1] P. T. Hoa, T. X. Dinh, and V. T. Dau, "Design Study of Multidirectional Jet Flow for a Triple-Axis Fluidic Gyroscope," IEEE Sens. J., vol. 15, no. 7, pp. 41034113, Jul. 2015.

[2] X. F. Leng, J. H. J. Y. Zhang, Y. Jiang, J. H. J. Y.
Zhang, X. C. Sun, and X. G. Lin, "Theory and experimental verification of spiral flow tube-type valveless piezoelectric pump with gyroscopic effect," Sensors Actuators, A Phys., vol. 195, pp. 1-6, 2013.

[3] G. Kock, P. Combette, B. Chariot, A. Giani, M. Schneider, and C. Gauthier-Blum, "Study and realization of a fluidic thermal gyrometer," in 2017 Symposium on Design, Test, Integration and Packaging of MEMS/MOEMS (DTIP), 2017, pp. 1-5.

[4] V. T. Dau, T. X. Dinh, C. D. Tran, P. N. Bui, D. D. Vien, and H. T. Phan, "Fluidic mechanism for dualaxis gyroscope," Mech. Syst. Signal Process., vol. 108, pp. 73-87, 2018.

[5] K. Tanaka, V. T. Dau, R. Sakamoto, T. X. Dinh, D. V. Dao, and S. Sugiyama, "Fabrication and Basic Characterization of a Piezoelectric Valveless Micro Jet Pump," Jpn. J. Appl. Phys., vol. 47, no. 11, pp. 8615-8618, Nov. 2008.

[6] V. T. Dau, T. X. Dinh, T. T. Bui, and C.-D. Tran, "Vortex flow generator utilizing synthetic jets by diaphragm vibration," Int. J. Mech. Sci., vol. 142143, 2018.

[7] V. T. Dau, T. Shiozawa, D. V. Dao, H. Kumagai, and S. Sugiyama, "A dual axis gas gyroscope utilizing low-doped silicon thermistor," in 18th IEEE International Conference on Micro Electro Mechanical Systems, 2005. MEMS 2005., 2005, pp. 626-629.

[8] N. T. Van et al., "An electrohydrodynamic gyroscope," Sensors Actuators A Phys., vol. 315, p. 112291, Nov. 2020.

[9] T. M. Dauphinee, "Acoustic Air Pump," Rev. Sci. Instrum., vol. 28, no. 6, p. 452, Dec. 1957.

[10]E. P. Mednikov, B. G. Novitskii, E. P. Mednikov, and B. G. Novitskii, "Experimental study of intense acoustic streaming," Akust. Zhurnal, vol. 21, pp. 245-249, 1975.

[11]N. T. Van et al., "A Circulatory Ionic Wind for Inertial Sensing Application," IEEE Electron Device Lett., vol. 40, no. 7, pp. 1182-1185, Jul. 2019.

[12] Y. Fukatsu, E. Nomura, and K. Matsu, "Gas rate gyro," US4941353, 1990.

[13] Y. Cai and Y. Zhao, "Ion discharge gyroscope," US8146423, 2012.

[14] M. Robinson, "Movement of air in the electric wind of the corona discharge," Trans. Am. Inst. Electr. Eng. Part I Commun. Electron., vol. 80, no. 2, pp. 143-150, 1961.

[15]P. Giubbilini, "The current-voltage characteristics of point-to-ring corona," J. Appl. Phys., vol. 64, no. 7, pp. 3730-3732, 1988.

[16] V. T. Dau, T. X. Dinh, C. D. Tran, T. T. Bui, and H. T. Phan, "A study of angular rate sensing by corona discharge ion wind," Sensors Actuators A Phys., vol. 277, pp. 169-180, Jul. 2018.

[17] OpenFOAM ${ }^{\circledR}$, “OpenFOAM ${ }^{\circledR} \mid$ The OpenFOAM Foundation," 2016. [Online]. Available: http://openfoam.org/.

\section{CONTACT}

*V. T. Dau; v.dau@griffith.edu.au.

**T. T. Bui; tungbt@,vnu.edu.vn. 